\title{
A JOINT APPROACH TO SAFETY, SECURITY AND RESILIENCE USING THE FUNCTIONAL RESONANCE ANALYSIS METHOD
}

\author{
Corinna Köpke ${ }^{I}$ \\ Jan Schäfer-Frey ${ }^{2}$ \\ Evelin Engler ${ }^{3}$ \\ Carl Philipp Wrede ${ }^{1}$ \\ Jennifer Mielniczek ${ }^{4,1}$ \\ 1) German Aerospace Centre, Institute for the Protection of Maritime \\ Infrastructures, Germany \\ 2) FICHTNER GmbH \& Co. KG, Germany \\ 3) German Aerospace Centre, Institute for Communications and Navigation, \\ Germany \\ 4) Jade University, Germany
}

\begin{abstract}
The protection of Offshore Wind Farms (OWF), a critical part of maritime infrastructure, faces new challenges due to the continually increasing share of renewable power generation (planned to reach $65 \%$ until 2030 in Germany). This is especially due to the large size of individual OWF (centralized generation units) and new threats such as climate change and their potential as targets for terrorism. It is no longer sufficient to simply optimize the performance of energy generation; the infrastructure also needs to be kept resilient when facing these new threats. To improve resilience, safety and security measures have to be taken into account and therefore safety, security and resilience (SSR) need to be addressed collectively.

To this end, SSR goals are identified for a generic OWF by analyzing stakeholder needs and expectations. These goals include not only safe energy generation but also environmental protection, compliance with regulations, hazard defense and security. The SSR goals are classified and detailed in (i) who/what needs protection, (ii) hazards, and (iii) measures with available sensors. A common modeling tool in resilience research, the Functional Resonance Analysis Method (FRAM) is employed to visualize and model interrelations/interactions between SSR goals. The feasibility to model SSR goals as functions and the respective expected variabilities with FRAM are also studied. Further, the possibility to identify critical paths in the FRAM model which allows the introduction of cascade effects is assessed. Critical SSR goals are identified that need further measures to increase the level of fulfillment and to keep the infrastructure protected.
\end{abstract}

Keywords: Infrastructure protection, Offshore Wind, FRAM

(C) 2019 Köpke, C., Schäfer-Frey, J., Engler E., Wrede C.P., and Mielniczek, J. This is an Open Access article distributed under the terms of the Creative Commons Attribution-NonCommercial 4.0 International License (http://creativecommons.org/licenses/by-nc/4.0), permitting all non-commercial use, distribution, and reproduction in any medium, provided the original work is properly cited.

ISBN: 978-91-88898-41-8

DOI: https://doi.org/10.15626/rea8.10 


\section{INTRODUCTION}

The offshore wind industry started in Germany back in 2010 with the commissioning of the first Offshore Wind Farm (OWF) "Alpha Ventus" consisting of 12 wind turbines $5 \mathrm{MW}$ installed capacity each [1]. To date (status March 2019) a total capacity of 6,617 MW is achieved with operation in the German North Sea and Baltic Sea. The German Federal Network Agency (Bundesnetzagentur reading as "BNetzA") expects that by end of 2020 the capacity of OWF will increase up to approximately 7,700 MW [2]. The political framework set out by the federal government of Germany aims to install and bring into operation OWF with a total capacity of 15,000 MW by 2030 [3]. In general, the typical maximum size of an offshore grid connection system constructed by the transmission operator is designed to carry $900 \mathrm{MW}$. Currently it is not foreseen that a significant change in the transmission grid connection systems to different capacities will occur.

Due to the increasing importance of the energy generated by offshore wind in Germany compared to the overall energy production, and considering the Ordinance on the Determination of Critical Infrastructures under the Federal Office for Information Security Act [4], the overall focus is put on the criticality of the energy sector in general. Electricity generating units, plants and systems to control or bundle electrical power above a threshold of $420 \mathrm{MW}$ net capacities are defined as critical infrastructure $[4,5]$. This leads to the conclusion that all offshore substation platforming with a capacity of $420 \mathrm{WM}$ and above will be part of the critical infrastructure and have to comply with [4]. Owners of such critical infrastructure are required to submit certificates relating to security for their relevant infrastructure [6]. This obligation indicates the increasing significance of security of electrical generation units and especially also the significance of its IT security.

Recent research undertaken with respect to safety, security and resilience of OWF has been published in various but very specific domains, such as, occupational safety [7], resilience of turbine maintenance [8], turbine structures [9], cyber security [10] and resilience towards hurricanes [11]. The following work is motivated by the current focus of research on specific aspects only together with changes of significance of the offshore wind industry as a part of the nation-wide electricity supply in Germany. Thus, the focus of research is expanded to a global approach integrating all relevant aspects of safety, security and resilience assessment in one modelling approach as further described in this paper.

Generally speaking, safety and security represent desired states of socio-technical systems, whereby the specific meaning of both has to be derived from the specific safety and security objectives pursued by owners, regulators, users, operators or other stakeholders. Examples, which are relevant for OWF, are the occupational safety, the functional safety, environmental protection and supply reliability. Operational safety addresses (i) the trouble-free functioning of the system (functional safety) and (ii) the absence of risks for human life (occupational safety), goods (value preservation) and environmental impact of the OWF itself (environmental protection). As an active component of the transnational power supply systems, the OWFs contribute to the energy supply reliability or may act as an interfering factor. A trouble-free functioning of OWF can be endangered by environmental impacts or unintended as well as intended anthropogenic interferences on OWF operation. Such interfering influences may be caused by surrounding air and sea traffic, by criminal or terroristic attacks or by disruptions and disturbances by supporting services needed to ensure a well-functioning of the OWF. The ability to withstand the latter depends on the system's resilience. A general definition provided by United Nations Office for Disaster Risk Reduction (UNISDR) describes resilience as the "ability of a system, community or society to resist, absorb, accommodate to and recover from 
the effects of a hazard in a timely and efficient manner" [12]. Applying this definition to OWF results in the requirements that they have to be able to absorb or adapt to typical disturbances, disruptions and changes. The fulfillment of these requirements is a general design criterion, but also a permanent challenge for efficient operation and effective maintenance strategies. These requirements focus on operational and functional safety of OWF in relation to known or expected failures and breakdowns. The UNISDR definition stresses also the need for broader capabilities such as the monitoring of boundary conditions of the currently operated system, the system handling nearby or outside the safety boundaries, and the efficient and effective system rebounding from disrupting or destroying events [13-15]. The need for broader capabilities results from uncertainties in modelling and design as well as from the threat of unanticipated safety and security violations.

To study the above outlined safety and security violations, simulations can be employed. To this end, complex socio-technical systems like OWF need to be modelled which can be challenging because of non-linear relations and the involvement of humans. The Functional Resonance Analysis Method (FRAM) allows the representation of the system in terms of processes and functions, instead of physical structure [18]. FRAM is based on four main principles. That is, (i) functions fail and succeed in the same way; (ii) the latter arises from performance variabilities of functions; (iii) variabilities of several functions can lead to nonlinear effects; and (iv) functional resonance is caused by unintended variability interactions of functions. For more details on the principles as well as the FRAM model visualizer (FMV) see [19]. FRAM has been applied to study performance variabilities and incidents in complex systems in different domains such as air traffic management [20,21], urban transport systems [22], environmental aspects of a sinter plant [23] and vessel traffic services [24]. Here, FRAM will be applied to model and visualize safety and security aspects in OWF.

In Section 2 the relation between safety, security and resilience is discussed, an approach to identify general SSR goals for OWF is presented and interrelations between the goals are studied. In Section 3 detailed SSR goal interrelations are modelled in FRAM, variabilities of functions are defined and an algorithm to study cascading effects and critical dependencies between functions based on the FRAM model is presented. In Section 4 our findings are summarized and an outlook to further research questions is given.

\section{THE JOINT APPROACH TO SAFETY, SECURITY AND RESILIENCE}

The discussion of safety and security aspects has to be based on the variety of dangers a system faces, differentiating between safety and security often results from the differentiation between unintended and intended dangers. For example, a loss of operational or functional safety of a system (e.g. OWF) may be induced by single component failures, excessive wear and tear, or poor controlling and careless operator decisions. However, the loss of functional safety may also be the primary target of criminal activities to e.g. destabilize the power supply of whole regions. In times of global terrorism and evolving threats by state sponsored actors most of the safety objectives cannot be ensured without sufficient consideration of security aspects [16]. It is known that the system's vulnerability against criminal and terroristic attacks can be decreased by the implementation of defense mechanisms in the endangered system. This can include means for protection, observation and intervention $[17,18]$. Functional and operational safety of protection, observation and intervention measures determine the success or non-success of averting a danger. The interactions between safety and security aspects illustrate the necessity of joint consideration of both during system design, operation and management [16].

The aspiration for resilient systems, communities or societies addresses the desire that these systems are able to function well and to meet the required performance. A general resilience 
doesn't exist. It is necessary to specify what "well-functioning within the required performance" means. For technological systems it may be sufficient that resilience aims at operational and functional targets. For complex, networked and socio-technical systems it has to be expected that resilience is related to different, sometimes contrary objectives. In both cases, however, an effective handling of safety and security aspects is an intrinsic task of all resilience concepts.

\subsection{Stakeholders and SSR goals}

To derive safety and security objectives, here generally called SSR goals, we identified stakeholders of the OWF namely the owner, operator, works manager, maintenance provider, logistic companies, grid connection, public authorities, coast guard, trade control, rescue forces, vessel and air traffic services, international organizations, insurance companies, investors, energy exchange market, media, society, environmental associations, fishery and shipping. All these stakeholders have interests with respect to the OWF which are linked to their activities in/ around the OWF. Thus, we analyzed the stakeholder tasks, identified their interests and extracted the objectives with relevance for safety and security. Further, we classified the objectives and derived nine general SSR goals, namely accident prevention (SSR1, avoidance of accidents between the plant and e.g. ships), security (SSR2, defense against e.g. attacks, vandalism), compliance (SSR3, respecting laws and regulations), occupational safety (SSR4, safety of people in the OWF), environmental protection (SSR5, protection of flora and fauna), reputation (SSR6, image of stakeholders), plant safety (SSR7, functioning of the OWF including operation and maintenance), supply reliability (SSR8, guarantee of energy supply) and finance (SSR9, monetary interests). Stakeholders have different interests and are therefore associated to different SSR goals. Thus, the safety and security level which depends on the degree of fulfillment of the SSR goals is based on different sets of SSR goals and varies between stakeholders.

\subsection{Interrelations between SSR goals}

The nine SSR goals described above are interrelated; this has been taken into account before modelling the processes and to sort SSR goals hierarchically. The dependencies are visualized in Figure 1 (left). As an example, accident prevention influences directly security and, compliance with respect to the regulation for the ease of shipping, occupational safety, environmental protection and plant safety. That is, a detection system for ships entering the safety zone of the OWF which will help to avoid collisions between ships and wind turbines but also to address security issues. Regulations for accident prevention need to be addressed to be compliant. Accidents could result in the harm of workers, in leakages that disturb the environment and in loss of functionality of the plant.

Regarding the interrelations, SSR goals were prioritized to extract those most relevant which will be the focus for the remainder of this work. To this end, the SSR goals were ranked highest, which had the most influence on other SSR goals because their fulfillment builds the basis for the fulfillment of other SSR goals. Thus, the four most important SSR goals are accident prevention, security, occupational safety and plant safety. However, environmental protection was also included in the further assessment as it represents a major concern for all life around the OWF.

\section{REPRESENTATION OF SSR GOALS IN FRAM}

\subsection{Detailed SSR goals in FRAM}


In the next step, we detailed the five overall SSR goals and described them in 64 functions for an arbitrary OWF. We distinguish in four categories of functions, namely overall SSR goals (see section 2.1), detailed SSR goals (function \# 1 to 21), SSR measures (function \# 22 to 53) and the analysis of sensor data (function \# 54 to 64). All functions are listed in Table 1.

\begin{tabular}{|c|c|c|c|c|c|c|c|c|c|}
\hline \# & Function name & $\mathbf{p}$ & $\mathbf{t}$ & f & \# & Function name & $\mathbf{p}$ & $\mathbf{t}$ & f \\
\hline $\mathbf{1}$ & Protect plants & $\mathrm{L}$ & $\mathrm{H}$ & $\mathrm{L}$ & 33 & Firefighting & $\mathrm{L}$ & $\mathrm{M}$ & $\mathrm{H}$ \\
\hline 2 & Protect water quality & $\mathrm{M}$ & $\mathrm{H}$ & $\mathrm{M}$ & 34 & Fire detection & $\mathrm{L}$ & $\mathrm{M}$ & $\mathrm{H}$ \\
\hline 3 & Protect whales & $\mathrm{L}$ & $\mathrm{M}$ & $\mathrm{L}$ & 35 & Safe transfer & $\mathrm{L}$ & $\mathrm{M}$ & $\mathrm{H}$ \\
\hline 4 & Protect fish & $\mathrm{L}$ & $\mathrm{M}$ & $\mathrm{L}$ & 36 & Measures helicopter & $\mathrm{L}$ & $\mathrm{H}$ & $\mathrm{H}$ \\
\hline 5 & Protect birds & $\mathrm{L}$ & $\mathrm{M}$ & $\mathrm{L}$ & 37 & Measures climbing & $\mathrm{M}$ & $\mathrm{H}$ & $\mathrm{M}$ \\
\hline 6 & Protect bats & $\mathrm{L}$ & $\mathrm{M}$ & $\mathrm{L}$ & 38 & Measures diving & $\mathrm{M}$ & $\mathrm{H}$ & $\mathrm{M}$ \\
\hline 7 & Safety plane & $\mathrm{L}$ & $\mathrm{H}$ & $\mathrm{H}$ & 39 & Rescue chain & $\mathrm{L}$ & $\mathrm{H}$ & $\mathrm{H}$ \\
\hline 8 & Safety helicopter & M & M & $\mathrm{H}$ & 40 & Telemedicine & $\mathrm{M}$ & $\mathrm{M}$ & $\mathrm{M}$ \\
\hline 9 & Safety ship & $\mathrm{M}$ & $\mathrm{M}$ & $\mathrm{H}$ & 41 & Shelter & $\mathrm{L}$ & $\mathrm{M}$ & $\mathrm{M}$ \\
\hline $\mathbf{1 0}$ & Safety ROV & $\mathrm{H}$ & $\mathrm{M}$ & $\mathrm{L}$ & 42 & Regular maintenance & $\mathrm{L}$ & $\mathrm{H}$ & $\mathrm{H}$ \\
\hline 11 & Safety submarine & $\mathrm{L}$ & $\mathrm{H}$ & $\mathrm{H}$ & 43 & Traffic control & $\mathrm{L}$ & $\mathrm{L}$ & $\mathrm{M}$ \\
\hline 12 & Protect foundation & $\mathrm{L}$ & $\mathrm{H}$ & $\mathrm{H}$ & 44 & Guard vessel & $\mathrm{M}$ & $\mathrm{M}$ & $\mathrm{L}$ \\
\hline 13 & Protect tower & $\mathrm{L}$ & $\mathrm{H}$ & $\mathrm{H}$ & 45 & PPE & $\mathrm{M}$ & $\mathrm{H}$ & $\mathrm{L}$ \\
\hline 14 & Protect rotor/ nacelle & M & $\mathrm{H}$ & $\mathrm{H}$ & 46 & Landing area & $\mathrm{M}$ & $\mathrm{M}$ & $\mathrm{L}$ \\
\hline 15 & Protect cable & $\mathrm{L}$ & $\mathrm{H}$ & $\mathrm{H}$ & 47 & Trainings & $\mathrm{L}$ & $\mathrm{M}$ & $\mathrm{L}$ \\
\hline 16 & Protect OSS & $\mathrm{L}$ & $\mathrm{H}$ & $\mathrm{H}$ & 48 & Decompression chamber & $\mathrm{L}$ & $\mathrm{M}$ & $\mathrm{L}$ \\
\hline 17 & Protect converter station & $\mathrm{L}$ & $\mathrm{H}$ & $\mathrm{H}$ & 49 & IT-security & $\mathrm{M}$ & $\mathrm{H}$ & $\mathrm{H}$ \\
\hline 18 & Safety of worker & $\mathrm{M}$ & $\mathrm{M}$ & $\mathrm{M}$ & 50 & Prevent espionage & $\mathrm{L}$ & $\mathrm{H}$ & $\mathrm{H}$ \\
\hline 19 & Shipwrecked men rescued & $\mathrm{L}$ & $\mathrm{M}$ & $\mathrm{L}$ & 51 & Repel attacks & $\mathrm{L}$ & $\mathrm{H}$ & $\mathrm{H}$ \\
\hline 20 & Safe communication & $\mathrm{M}$ & $\mathrm{L}$ & $\mathrm{M}$ & 52 & Avoid manipulation & $\mathrm{L}$ & $\mathrm{H}$ & $\mathrm{H}$ \\
\hline 21 & Safe data & $\mathrm{L}$ & $\mathrm{M}$ & $\mathrm{M}$ & 53 & Access control & $\mathrm{L}$ & $\mathrm{H}$ & $\mathrm{H}$ \\
\hline 22 & Avoid pollutants & $\mathrm{L}$ & $\mathrm{M}$ & $\mathrm{H}$ & 54 & Observe leakage & $\mathrm{L}$ & $\mathrm{M}$ & $\mathrm{M}$ \\
\hline 23 & Bubble curtain & $\mathrm{L}$ & $\mathrm{M}$ & $\mathrm{L}$ & 55 & Observe water quality & $\mathrm{L}$ & $\mathrm{M}$ & $\mathrm{M}$ \\
\hline 24 & Observe population & $\mathrm{L}$ & $\mathrm{L}$ & $\mathrm{L}$ & 56 & AIS & $\mathrm{L}$ & $\mathrm{M}$ & $\mathrm{H}$ \\
\hline 25 & Avoid collision plant/animal & $\mathrm{M}$ & $\mathrm{M}$ & $\mathrm{M}$ & 57 & Weather data & $\mathrm{M}$ & $\mathrm{L}$ & $\mathrm{M}$ \\
\hline 26 & Collision avoidance & $\mathrm{L}$ & $\mathrm{H}$ & $\mathrm{M}$ & 58 & Heat detection & $\mathrm{L}$ & $\mathrm{L}$ & $\mathrm{H}$ \\
\hline 27 & Sonar transponder & $\mathrm{L}$ & $\mathrm{M}$ & $\mathrm{M}$ & 59 & Smoke detection & $\mathrm{L}$ & $\mathrm{L}$ & $\mathrm{H}$ \\
\hline 28 & Warning lights & $\mathrm{M}$ & $\mathrm{M}$ & $\mathrm{M}$ & 60 & CMS & $\mathrm{L}$ & $\mathrm{M}$ & $\mathrm{M}$ \\
\hline 29 & Weather measures & $\mathrm{M}$ & $\mathrm{L}$ & $\mathrm{M}$ & 61 & EPIRB & $\mathrm{L}$ & $\mathrm{L}$ & $\mathrm{H}$ \\
\hline 30 & UXO clearance & $\mathrm{L}$ & $\mathrm{H}$ & $\mathrm{H}$ & 62 & People tracking & $\mathrm{L}$ & $\mathrm{L}$ & $\mathrm{M}$ \\
\hline 31 & Avoid technical failure & $\mathrm{M}$ & $\mathrm{M}$ & $\mathrm{M}$ & 63 & CCTV & $\mathrm{M}$ & $\mathrm{L}$ & $\mathrm{M}$ \\
\hline 32 & Lightning protection & $\mathrm{L}$ & $\mathrm{H}$ & $\mathrm{H}$ & 64 & PLB & $\mathrm{M}$ & $\mathrm{L}$ & $\mathrm{L}$ \\
\hline
\end{tabular}

Table 1: List of all FRAM functions with respective probability of failure $p$, time to restore $t$ and influencing factor $f$. Values are either low $(L)$, medium $(M)$ or high $(H)$; see text for details. ROV (Remotely Operated Vehicle), OSS (Offshore Sub-Station), UXO (Unexploded Ordnance), PPE (Personal Protection Equipment), AIS (Automated Identification System), CMS (Condition Monitoring System) EPIRP (Emergency Position-Indicating Radio Beacon), CCTV (Close-Circuit TeleVision), PLB (Personal Life Beacon).

Each sensor data analysis controls and supervises SSR measures. SSR measures can be time controlled by other measures (executed one after the other) and precondition or resource for another SSR measure (needed or mandatory for the execution). The specific function type, 'detailed SSR goal' is required for the fulfillment of certain overall SSR goals namely accident 
prevention (function \# 7 to 11), security (function \# 15 to 18 and 20 to 21), occupational safety (function \# 18 to 19), environmental protection (function \# 1 to 6), and plant safety (function \# 12 to 17). Each function holds a certain probability to fail/ to not perform as desired, a certain time that it takes to restore/ repair the function and a certain factor with which it influences downstream functions. The probability of failure $\mathrm{p}$ was assessed as low $(\mathrm{L}, 0.005)$, medium $(\mathrm{M}$, $0.015)$ and high $(H, 0.02)$. The time to recover $t$ can either be low ( $L$, between 2 and 5 days), medium (M, between 6 and 30 days) and high ( $\mathrm{H}$, between 30 and 50 days). The factor to influence downstream functions f has been chosen to be low $(\mathrm{L}, 1.05)$, medium $(\mathrm{M}, 1.2)$ or high $(\mathrm{H}, 1.35)$.

The relation between the 64 functions is visualized in Figure 1 (right). The matrix contains all factors with which sensors and SSR measures influence downstream functions (SSR measures and detailed SSR goals) in case of failure. This matrix results from the developed FRAM model that visualizes possible connections between functions. The representation of functional interactions in a matrix has been applied to accident analysis with respect to tsunamis [25] and air traffic management [26] in a so called resilience analysis matrix (RAM).
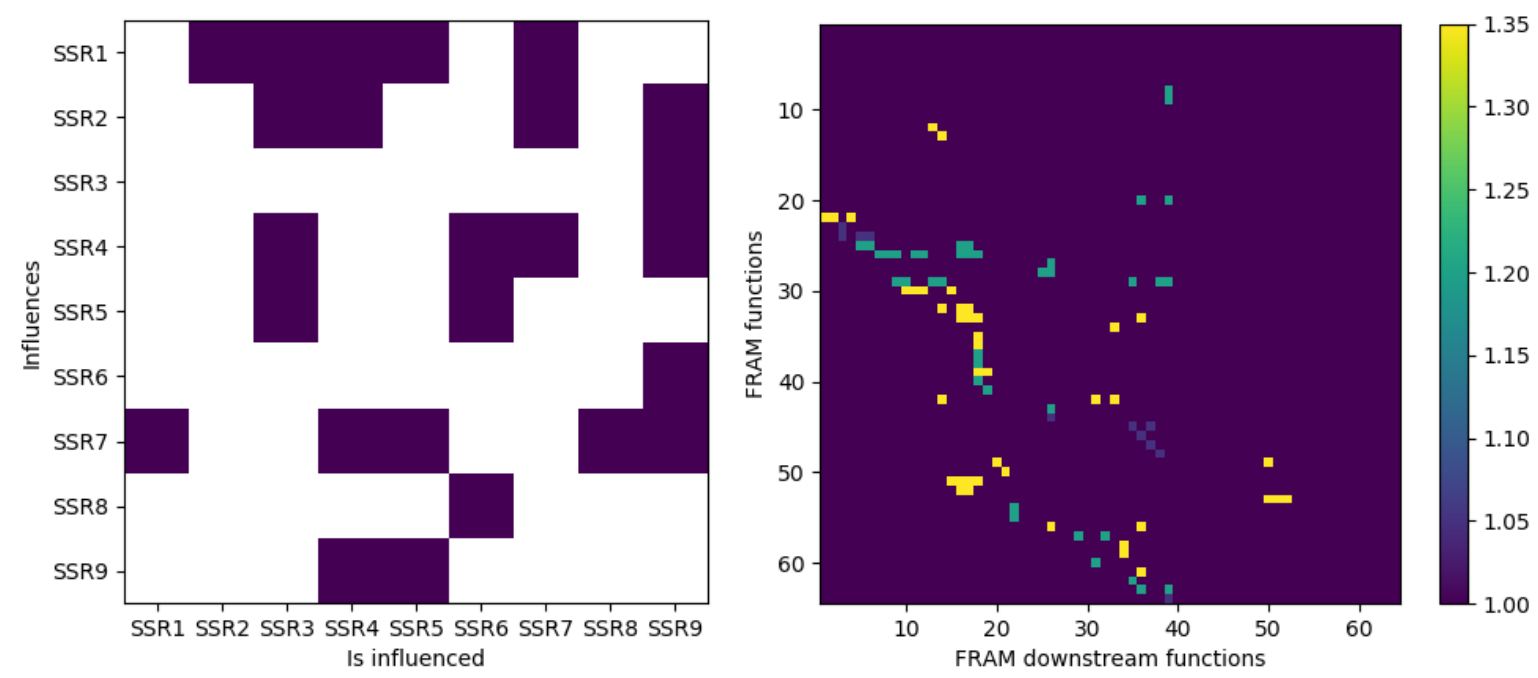

Figure 1: Visualization of functional interactions between overall SSR goals (graphic on the left) and the 64 functions presented in Table 1 (graphic on the right). In the left graphic, white implies 'no influence' and black 'influence'. In the right graphic, the color code gives the influencing factor $f$ whereby 1 implies 'no influence' and 1.35 'large influence'.

Note, that the list of functions considered is not complete and the level of abstraction is not always consistent for each overall SSR goal which means that for some SSR goals more influencing functions are specified than for others. This list and matrix do not replace a classical risk assessment but they could be enriched and improved by this approach and by the validation through stakeholders. The basic probability of failure for each function is assessed for the case where every implemented SSR measure of the OWF is functioning and thus represents the mean frequency of occurrence over a certain period of time. The time it takes to recover is representative of the affected function and not for all possible consequences in case of an incident. In the following, the approach and general methodology to identify critical function interactions and to evaluate the implementation of new functions will be presented.

\subsection{Propagate variabilities in FRAM}


The list (Table 1) and matrix (Figure 1, right) based on the FRAM model were introduced into a Monte Carlo simulation, comparable to an application presented in air traffic management [20]. The algorithm used to generate synthetic data by drawing random numbers is given below (Algorithm 1) and is implemented in Python. In principal, the algorithm iterates for $\mathrm{n}=365$ days and draws every day a random number a $(F)$ for each of the $\mathrm{nf}=64$ functions $\mathrm{F}$. If the random number is below the failure probability assigned in Table 1, the function fails. In the latter case, the failure probability p' $\left(\mathrm{F}_{\text {down }}\right)$ of downstream functions is multiplied with the factor $\mathrm{f}(\mathrm{F})$ given in Table 1 and the probability of failure for the broken function p'(F) is set to zero while it is restored (Lines 8 and 9 in Algorithm 1). For broken functions a countdown $b(F)$ is implemented until the function is restored with respect to the repair times $t(F)$ assigned in Table 1. Variabilities in restore time are based on a uniform distribution with upper and lower bound given in section 3.1 to represent delays in repair due to weather influence. The process of restoration involves the probability of failure being set to the original value $p(F)$ and then multiplied by all factors from broken upstream functions $\mathrm{f}\left(\mathrm{F}_{\mathrm{up}}\right)$.

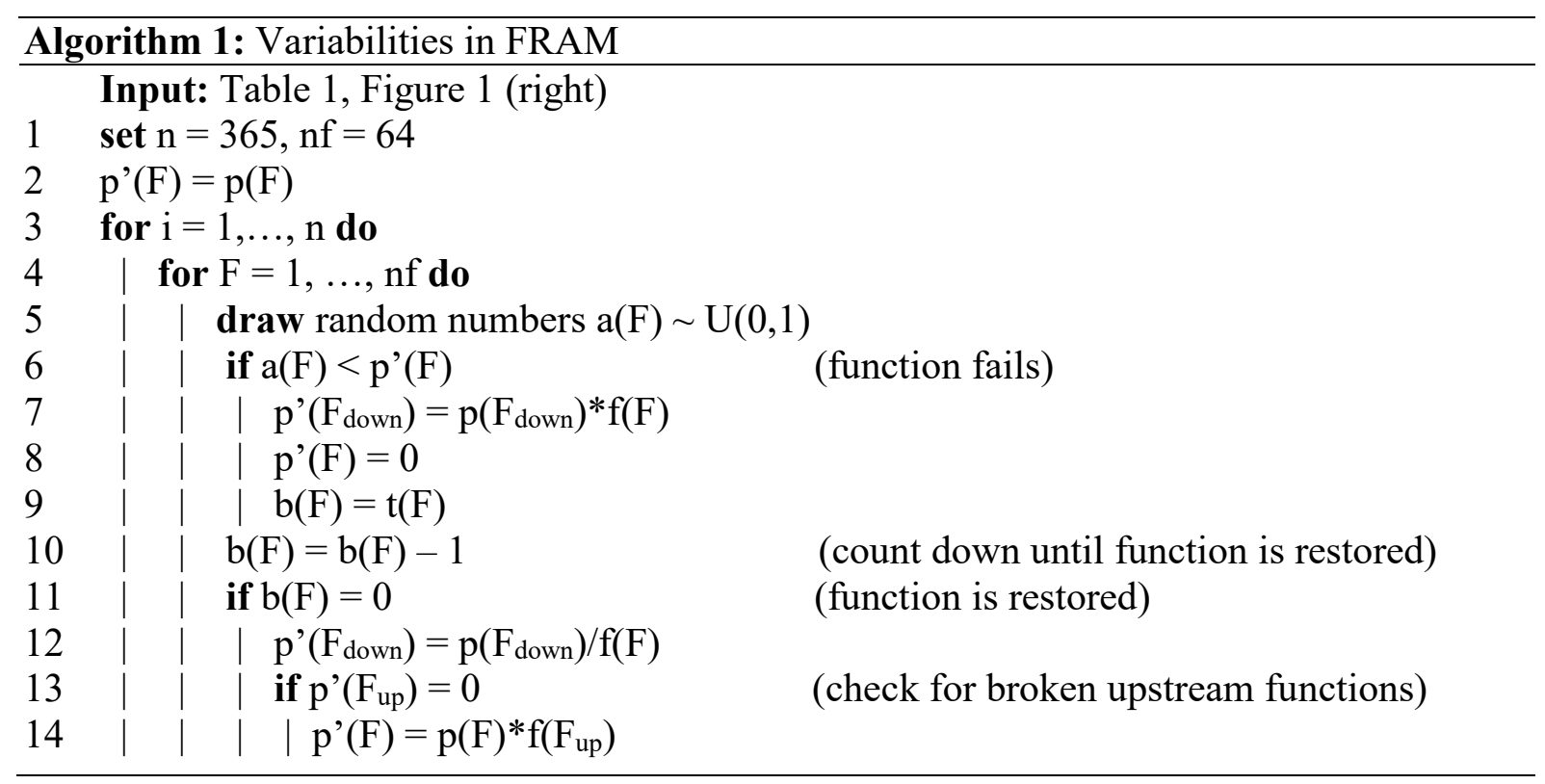

For simplicity, here functions can only work as desired or fail and in case of failure the failure probability of downstream functions is amplified. This will to be adapted in future work to reflect the idea of Safety-II, that function performance varies and that the behavior of several varying functions can amplify or damp the effects on downstream functions [e.g. 25]. The results for one run of 365 days are visualized in Figure 2. Failures of functions are marked with a failure probability of zero. Apparently, the largest probability of failure occurs for function \# 18 which is the detailed SSR goal 'Safety of worker'. To describe one example, after around 150 days the failure probability for this specific function increases continuously which is related to the failure of several upstream functions. These failures accumulate and finally lead to the failure of function \# 18 in day 170. The corresponding sequential function failures are shown as a branch of the large FRAM model in Figure 3. In this model the 'Safety of worker'-function is particularly fragile as it directly and indirectly depends on many upstream functions in comparison to the other detailed SSR goals. 


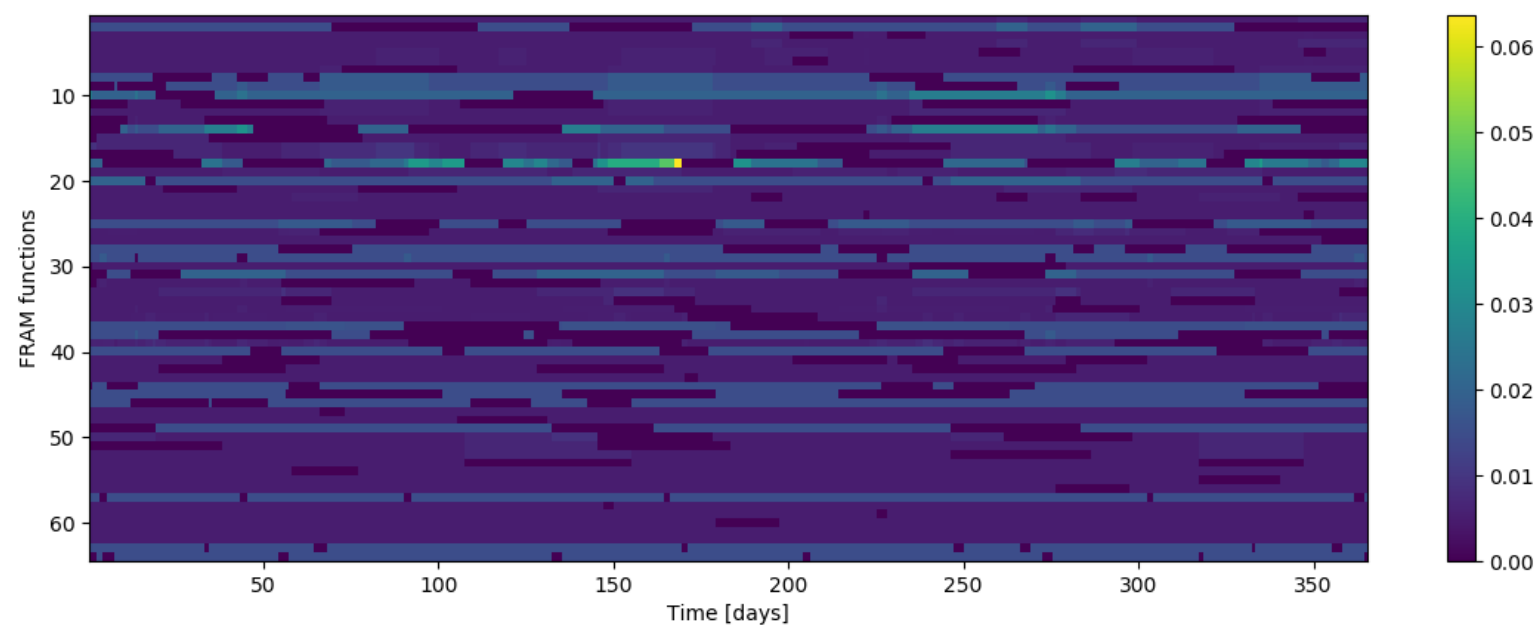

Figure 2: Failure probability for all FRAM functions during a simulation of 365 days.

Algorithm 1 is repeated 500 times to gain statistical insight into the distribution of failures of function \# 18 over one year. The result is presented in Figure 4 (left). The mean of the distribution lies at 6 and varies between 2 and 10 failures per year.

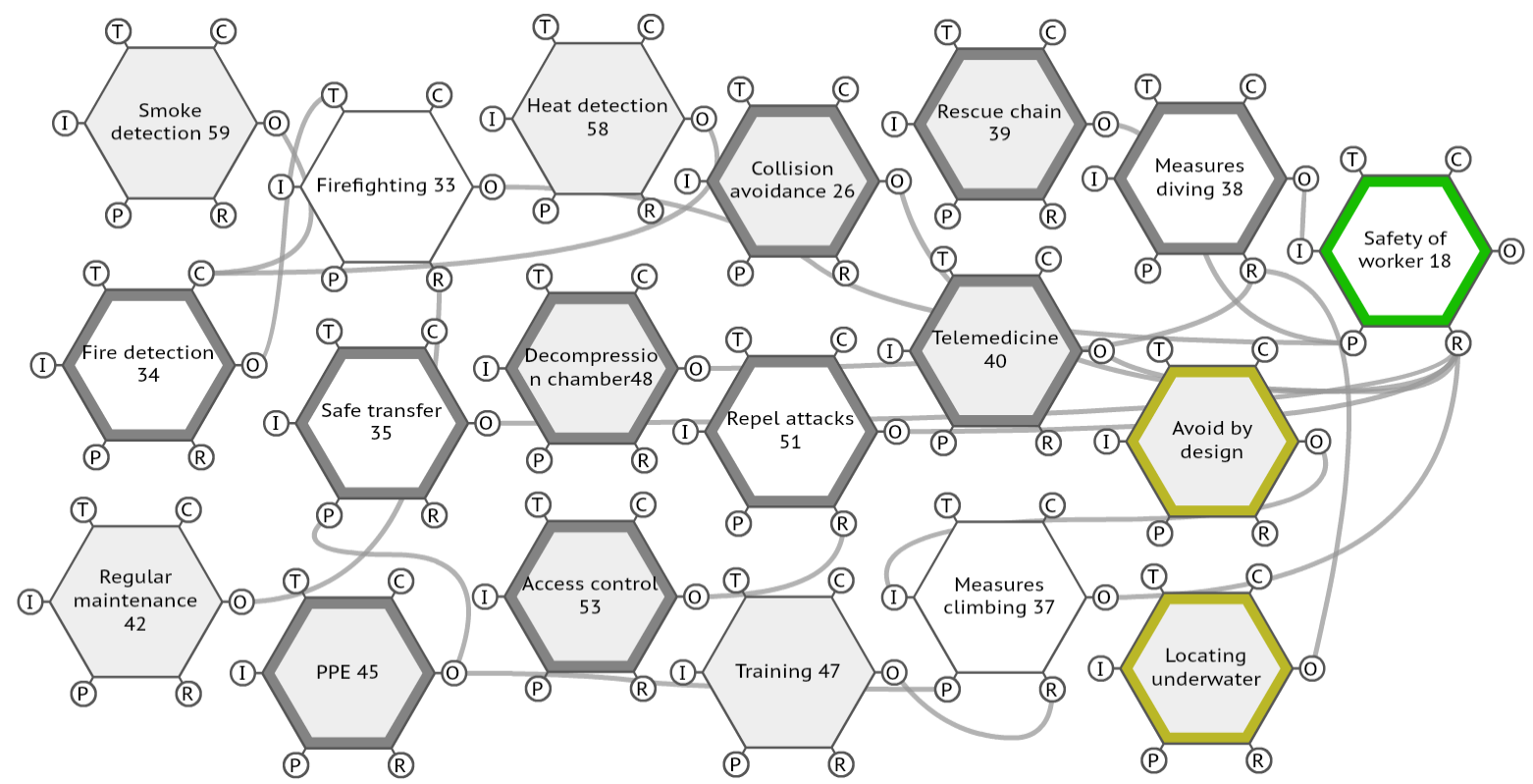

Figure 3: Visualization of the FRAM functions using the FMV. Broken functions between day 150 and 170 with respect to the example from Figure 3 are marked in grey. Yellow functions are additional measures that were not included in the simulation to generate Figure 2.

In order to decrease the number of failures of function \# 18 per year, we studied the case where more SSR measures are added to the FRAM model. We introduced two new functions namely 'Locating underwater' to reduce the probability of failure of 'Measures diving' and 'Avoid by design' to reduce the probability of failure of 'Measures climbing' (see the yellow functions in Figure 3). The influence of these two new functions depends on the adaptation of the whole model. If the failure probabilities of adjacent functions are not adapted, more upstream functions will naturally lead to more failures. However, here we adapt the influence of 'Measures climbing' on function \# 18 because the measure to avoid most diving will reduce 
the influence. This adaptation requires a general formalism which will be developed in future work.
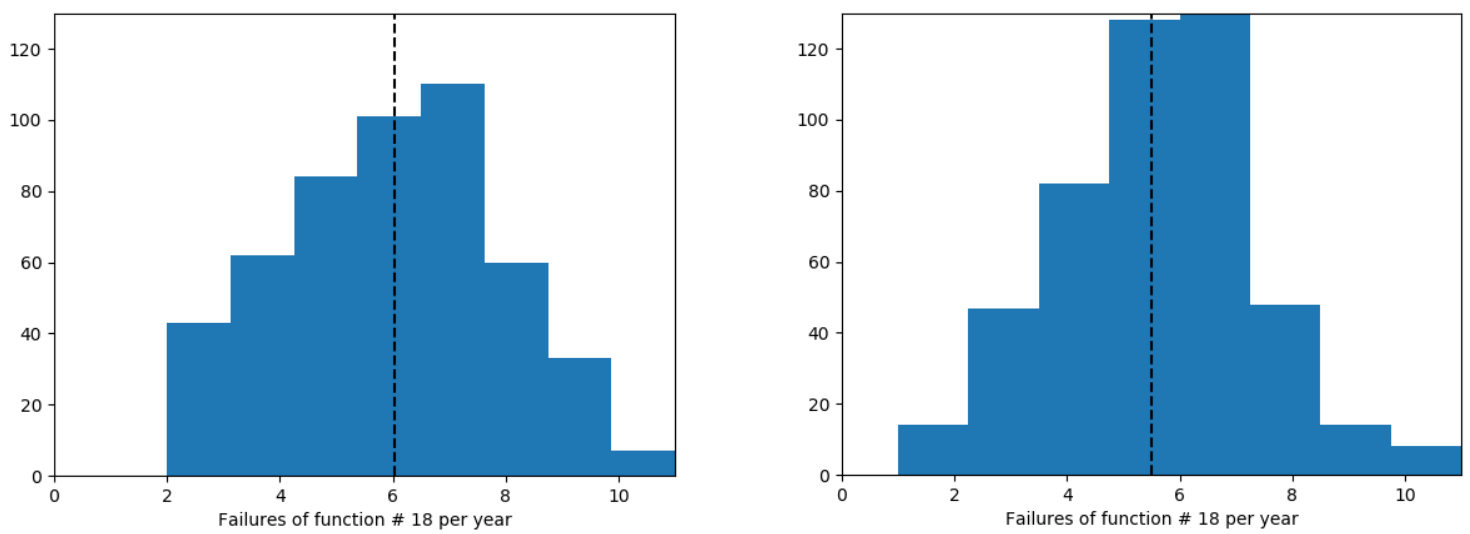

Figure 4: Histograms and mean (black dashed line) of failures of function \# 18 per year (365 days) repeated for 500 runs (years). Left graphic is based on the FRAM model with 64 functions presented partly in Figure 4. The right graphic is based on the FRAM model that contains two additional functions to reduce the failure probability for function \# 18.

The simulation results for 365 iterations and 500 runs including the two new functions are presented in Figure 4 (right). The mean is shifted towards 5 failures of function \# 18 per year. Apparently in such a complex system as an OWF, even if it is not modelled in every detail, new SSR measures lead to small but visible changes, resulting in a quantitatively proven safer work environment.

\section{CONCLUSION}

The paper shows the potential of FRAM-based simulations for the identification of interrelations and dependencies of SSR goals. This has been achieved by following a stringent approach of linking stakeholders with specific SSR goals, following a quantified description and analysis of these and finally by the development of a simulation implementation to propagate function failures through the FRAM model.

The identification of SSR goals revealed the differences in priority and relevance of specific goals depending on the stakeholder's context. The chosen method to select SSR goals for detailed analysis requires further validation especially in the context of real infrastructures. However, it appears legitimate as initial approach with a focus on demonstrating the concept of a simulation-based identification of critical SSR goals for complex systems like OWFs. The description of SSR goals as quantifiable functions is consistent with current risk management approaches of infrastructure operators and will be improved with the involvement of stakeholders.

It is noteworthy that effects like cascading behavior have been observed in the simulation in the expected manner. This may allow for predictive detection of safety or security sensitive elements in infrastructures, with the potential for the system to be used by stakeholders in risk management. In further research the slope of failure probabilities of specific functions will be analyzed to potentially predict function failures. 
This research was able to show in principle an approach to model the safety, security and resilience aspects of complex systems. The numbers assigned to functions in this paper will still differ from those relating to a real functioning OWF as the risk assessment and FRAM model are a work in progress. A further validation of these values is required in order to determine the strengths and weaknesses of the method and to ensure a correct replication of interactions.

The reevaluation of the whole system's fulfillment level of SSR goals after a modification or addition of a specific measure revealed a potential application of the tool in the field of risk management practices. Isolated revisions of processes or implementations of safety or security mechanisms are often not triggering the reevaluations of the whole system due to resource constrains. This method allows a review of effects which include the interaction between various SSR goals, which has the potential to gain a better understanding of the effectiveness of changes. However, the current approach is limited to two conditions (failure and success) and could be extended to a model that represents a wider performance variability, as this should describe a realistic systems behavior and would integrated a Safety-II understanding of operations. Further research will be undertaken to evaluate if the demonstrated method can be applied for general infrastructure optimization and if it has the potential to increase resilience against failures and interruptions.

\section{REFERENCES}

[1] FACT-SHEET alpha ventus. (2015). https://www.alpha-ventus.de/ueberblick/\#top

[2] Bundesnetzagentur. (2019).

https://www.bundesnetzagentur.de/DE/Sachgebiete/ElektrizitaetundGas/Unternehmen_Institu tionen/Ausschreibungen/Offshore/offshore-node.html

[3] Bundesministerium für Wirtschaft und Energie. (2019).

https://www.bmwi.de/Redaktion/DE/Dossier/erneuerbare-energien.html

[4] Bundesministerium der Justiz und für Verbraucherschutz. (2017). https://www.gesetze-

im-internet.de/bsi-kritisv/BJNR095800016.html

[5] Bundesamt für Sicherheit in der Informationstechnik. (2017).

https://www.bsi.bund.de/SharedDocs/Downloads/DE/BSI/Publikationen/Broschueren/SchutzKritischer-Infrastrukturen-ITSig-u-UP-KRITIS.pdf?_blob=publicationFile\&v=7

[6] Bundesnetzagentur. (2018). IT-Sicherheitskatalog gemäß § 11 Absatz 1b

Energiewirtschaftsgesetz

https://www.bundesnetzagentur.de/SharedDocs/Downloads/DE/Sachgebiete/Energie/Unterne hmen_Institutionen/Versorgungssicherheit/IT_Sicherheit/IT_Sicherheitskatalog_2018.pdf? blob $=$ publicationFile $\& v=4$

[7] Lloyd, P.O. 19 - Health and safety of offshore wind farms, (2016) Offshore Wind Farms, Woodhead Publishing, Pages 573-587, https://doi.org/10.1016/B978-0-08-100779-2.00019-2.

[8] Mentes, A., \& Turan, O. (2018). A new resilient risk management model for Offshore Wind Turbine maintenance. Safety Science. https://doi.Org/10.1016/j.ssci.2018.06.022

[9] Wilkie, D., \& Galasso, C. (2017). Towards Resilient Offshore Wind Farms. In:

Titheridge, $\mathrm{H}$ and Parikh, P, (eds.) Selected Conference Proceedings: 3rd International Conference on Urban Sustainability and Resilience. (pp. pp. 117-129). UCL Centre for Urban Sustainability and Resilience: London, UK. http://discovery.ucl.ac.uk/10062559/

[10] Staggs, J., Ferlemann, D., \& Shenoi, S. (2017). Wind farm security: attack surface, targets, scenarios and mitigation. International Journal of Critical Infrastructure Protection, 17, 3-14. http://dx.doi.org/10.1016/j.ijcip.2017.03.001 
[11] Hartman, L. (2018). Wind Turbines in Extreme Weather: Solutions for Hurricane Resiliency. Office of Energy Efficiency \& Renewable Energy.

https://www.energy.gov/eere/articles/wind-turbines-extreme-weather-solutions-hurricaneresiliency

[12] United Nations Office for Disaster Risk Reduction (UNISDR) (2009): UNISDR terminology on disaster risk reduction

http://www.unisdr.org/files/7817_UNISDRTerminologyEnglish.pdf

[13] Woods, D. D. Essential characteristics of resilience. In E. Hollnagel, D.D. Woods and N.

Leveson (eds), Resilience Engineering: Concepts and precepts. Aldershot: Ashgate Publishing limited, page 21-24.

[14] Jackson, S., \& Ferris, T. (2012). Resilience Principles for Engineered Systems. Systems Engineering. The Journal of the International Council on Systems Engineering. Wiley

Periodicals Inc. DOI 10.1002/sys.21228.

[15] Woods, D. (2015). Four concepts for resilience and the implications for the future of resilience engineering. Reliability Engineering \& System Safety. 141.

10.1016/j.ress.2015.03.018.

[16] Beyerer, J., \& Geisler, J. (2016). A Framework for a Uniform Quantitative Description of Risk with Respect to Safety and Security. European Journal for Security Research, 1(2), 135150.

[17] Lichte, D., \& Wolf, K.-D. (2017). Quantitative multiple-scenario vulnerability assessment applied to a civil airport infrastructure. The 2nd International Conference on Engineering Sciences and Technologies. DOI: 10.1201/9781315210469-155

[18]Hollnagel, E., (2012). FRAM: The Functional Resonance Analysis Method: Modelling Complex Socio-technical Systems. Ashgate.

[19] Hollnagel, E. (2014). The Four Basic Principles of the FRAM.

http://functionalresonance.com/basic-principles.html

[20]Patriarca, R., Di Gravio, G., \& Costantino, F. A Monte Carlo evolution of the Functional Resonance Analysis Method (FRAM) to assess performance variability in complex systems, Safety Science, Volume 91, January 2017, Pages 49-60, ISSN 0925-7535, http://dx.doi.org/10.1016/j.ssci.2016.07.016.

[21] Herrera, I. A., \& Woltjer, R. (2010). Comparing a multi-linear (STEP) and systemic (FRAM) method for accident analysis. Reliability Engineering \& System Safety, 95(12), 1269-1275. doi:10.1016/j.ress.2010.06.003

[22] Bellini, E., Nesi, P., Pantaleo, G., \& Venturi, A. (2016). Functional resonance analysis method based-decision support tool for urban transport system resilience management. In 2016 IEEE International Smart Cities Conference (ISC2) (pp. 1-7). IEEE.

[23] Patriarca, R., Di Gravio, G. Costantino, F, \& Tronci, M. (2017). The Functional Resonance Analysis Method for a systemic risk based environmental auditing in a sinter plant: A semi-quantitative approach, Environmental Impact Assessment Review, Volume 63, Pages 72-86, ISSN 0195-9255, DOI: 10.1016/j.eiar.2016.12.002.

[24] Praetorius, G., Hollnagel, E., \& Dahlman, J. (2015). Modelling Vessel Traffic Service to understand resilience in everyday operations. Reliability engineering \& system safety, 141, 10-21. http://dx.doi.org/10.1016/j.ress.2015.03.020i

[25] Patriarca, R., Del Pinto, G., Di Gravio, G. \& Costantino, F. (2018). FRAM for Systemic Accident Analysis: A Matrix Representation of Functional Resonance, International Journal of Reliability, Quality and Safety Engineering, Vol. 25, No. 1, 1850001. DOI: $10.1142 / \mathrm{S} 0218539318500018$

[26] Lundberg, J. \& Woltjer, R. (2013) The Resilience Analysis Matrix (RAM): Visualizing functional dependencies in complex socio-technical systems. In 5th symposium on resilience engineering managing trade-offs (p. 103). 